\title{
The Therapeutic Potential of Conditioned Medium from Human Breast Milk Stem Cells in Treating Spinal Cord Injury
}

\author{
Maryam Borhani-Haghighi ${ }^{1}$, Shadan Navid ${ }^{2}$, Yousef Mohamadi ${ }^{3}$ \\ ${ }^{1}$ Department of Anatomy, School of Medicine, Tehran University of Medical Sciences, Tehran, Iran \\ ${ }^{2}$ Department of Anatomy, Gonabad University of Medical Sciences, Gonabad, Iran \\ ${ }^{3}$ Department of Anatomy, School of Medicine, Ilam University of Medical Sciences, Ilam, Iran
}

\section{Study Design: Experimental animal study.}

Purpose: This study investigated the therapeutic effects of human breast milk stem cell (BMSC)-conditioned medium (BMSC-CM) in a model of spinal cord injury (SCI) in male Sprague-Dawley rats.

Overview of Literature: $\mathrm{SCl}$ is one of the leading causes of disability in addition to sensory and motor impairment. So far, there have been no successful treatments for SCl. Given the positive outcomes associated with using stem cells and their derivatives as a treatment for various diseases, there is a growing interest in using them as an $\mathrm{SCl}$ treatment. Recent research has demonstrated that CM from stem cells has therapeutic advantages.

Methods: Human BMSCs were isolated and characterized, and CM was subsequently collected. Animals received an intrathecal administration of BMSC-CM after SCI. The activity of caspase-3 was measured to assess apoptosis, and levels of tumor necrosis factor- $\alpha$ and interleukin- $1 \beta$ were measured to assess inflammation. Also, sensory and locomotor performances were assessed after SCI and BMSC-CM administration.

Results: Administration of CM from BMSC reduced apoptosis and inflammation at the site of injury in a rat model of $\mathrm{SCI}(p<0.05)$. Motor, sensory, locomotor, and sensorimotor performances were significantly improved in rats that received BMSC-CM after SCI. Conclusions: Intrathecal administration of BMSC-CM improved recovery in a rat model of SCI.

Keywords: Conditioned media; Spinal cord injuries; Breast milk; Stem cells

\section{Introduction}

Spinal cord injury (SCI) is a serious neurological condition that impacts a patient's ability to function [1]. SCI is a result of the primary injury, which occurs as a direct consequence of trauma and the secondary injury, which occurs following the onset of reactive processes such as inflammation, ischemia, free radical production, apoptosis, and necrosis in the spinal cord [2]. SCI may lead to unpleasant complications, including various neurological problems such as the loss of sensory and motor function, autonomic nervous system dysfunction, pain, and ulcerous lesions [3]. These issues underscore the need to develop better strategies for the treatment of SCI [4]. With

Received Jan 22, 2019; Revised May 25, 2019; Accepted Jul 5, 2019

Corresponding author: Maryam Borhani-Haghighi

Department of Anatomy, School of Medicine, Tehran University of Medical Sciences, Poursina Street, Tehran, Iran

Tel: +98-21-66419072, Fax: +98-21-66419072, E-mail: borhanihm@gmail.com 
regard to these pathophysiological features, diverse treatment approaches have been suggested for SCI [5]. Previous studies have mainly focused on controlling and limiting the mechanisms involved with protecting the nerves against secondary damage in the affected area [6]. Replacing lost neurons and promoting neuronal regeneration using stem cells and their derivatives, such as stem cellconditioned medium (CM), has been extensively studied $[7,8]$. Breast milk is a valuable and noninvasive source of heterogeneous stem cells. As a result, it has received considerable attention in the field of regenerative medicine [9]. A portion of the breast milk expresses markers for mesenchymal stem cells (MSCs) [10]. Interestingly, a subpopulation of nestin-positive cells has also been reported in breast milk cells [11]. Nestin is a marker for neural stem cells. Differentiation of this easily-available source of stem cells into a neural cell lineage has been previously reported [12].

Although positive outcomes have been reported using stem cell therapy, there are still some disadvantages associated with these cells. For instance, the administration of stem cells may provoke an immune response or the formation of tumors [13]. Using cell-derived CM is an effective approach that may overcome these problems associated with stem cell administration [14]. Recent studies have revealed the neuroprotective effects of MSC-derived CM [15]. It has also been reported that CM from MSCs may improve neuronal survival and promote neurite extension [16]. However, there have been no studies conducted on the administration of breast milk stem cell (BMSC)-CM for SCI treatment. For this study, we examined the protective effects of BMSC-CM in a rat model of SCI.

\section{Materials and Methods}

\section{Experimental design}

Adult male Sprague-Dawley rats (250-280 g) were divided randomly into the following groups ( $\mathrm{n}=6$ /group). (1) Control group: laminectomy was performed; (2) SCI/Sham group: Dulbecco's Modified Eagle Medium (DMEM)/F12 culture medium was injected intrathecally into the injured site after the induction of SCI; and (3) BMSC-CM group: BMSC-CM was injected intrathecally into the site of injury after the induction of SCI induction. This study's protocol was reviewed and approved by the university (REC.1393.998).

\section{Isolation of cells from breast milk sample and char- acterization}

Breast milk was collected from healthy, lactating women who had given their consent prior to collecting samples. The samples were immediately transferred to the laboratory, diluted with an equal volume of sterile phosphatebuffered saline (PBS), and centrifuged at $15^{\circ} \mathrm{C} / 1,000 \times \mathrm{g}$ for 15 minutes. The pellets were washed thrice with PBS, and the isolated cells were cultured on gelatin-coated plates in DMEM/F12 culture medium supplemented with $10 \%$ fetal bovine serum and $1 \%$ glutamine. The cultured plates then were incubated at $5 \% \mathrm{CO}_{2}$ and $37^{\circ} \mathrm{C}$. The $\mathrm{CD}$ markers of the cultured cells were evaluated by flow cytometry after the third passage. The harvested cells were permeabilized and blocked using a solution containing Tween-20 and normal goat serum. Then, the cells were incubated with FITC (fluorescein isothiocyanate)-conjugated antiCD144, CD44, per-CP-conjugated anti-CD105, and PE (phycoerythrin)-conjugated anti-CD34 antibodies (all from Abcam). The cells were fixed using $4 \%$ paraformaldehyde, and the number of positive cells was measured using flow cytometry. The results were depicted as graphs created using FlowJo software (FlowJo LLC, Ashland, OR, USA) [17].

\section{Preparation of breast milk stem cell-conditioned me- dium}

BMSCs in the third passage, with approximately $80 \%$ of confluency, were washed with PBS, and the serum-free DMEM/F12 culture medium was added to the cells. After 48 hours, the BMSC-CM was collected and centrifuged at $3,000 \mathrm{rpm}$ for 10 minutes. The supernatant was concentrated 10 times using ultrafiltration using a $30 \mathrm{kDa}$ cutoff filter (Millipore, Burlington, MA, USA) and stored at $-80^{\circ} \mathrm{C}$ until use [18].

\section{Spinal cord injury induction}

The compression model of SCI was performed in this study. Rats were anesthetized with ketamine $(80 \mathrm{mg} / \mathrm{kg})$ and xylazine $(10 \mathrm{mg} / \mathrm{kg})$. Briefly, a laminectomy was made from T5 to T9 of the vertebral column. After that, the spinal cord was compressed at the level of T7 using a $20 \mathrm{~g}$ aneurysm clip for 1 minute. Following compression, the skin was sutured, and the rats received postoperative care 
for pain relief and to prevent infection [19]. At 48 hours after the induction of SCI, the prepared CM $(3 \mu \mathrm{L})$ was injected intrathecally into the site of injury using a 33-gauge Hamilton syringe. The bladder was manually drained daily until spontaneous bladder function returned.

\section{Locomotor test}

For evaluation of locomotor performance, the Basso, Beattie, and Bresnahan (BBB) open-field test was performed weekly for 6 weeks by a blinded examiner for each rat. Body, tail, and paw position, hind limb movement, trunk stability, and toe clearance were measured during the $\mathrm{BBB}$ test to measure locomotor ability. The $\mathrm{BBB}$ score ranged from 0 to 21; a score of 0 indicates no observable movement, whereas a score of 21 indicates no impairment in locomotor activity.

\section{Sensory test}

The cold allodynia test was used to evaluate sensory performance. Withdrawal of the foot in response to contact with acetone was recognized as a cold sensation. All rats were examined once per week for up to 6 weeks. Before starting the experiment, the animals were allowed to adapt to the testing apparatus for 10 minutes. A single drop of fresh acetone was dropped on the plantar surface of both feet. This procedure was repeated 5 times, with intervals of at least 5 minutes. Removing or shaking the leg was considered a positive reaction. The time taken to respond to the stimulus was measured for each animal.

\section{Assessment of caspase-3 activity for evaluation of apoptosis}

The level of apoptosis may be evaluated by measuring caspase- 3 activity. In this study, we used the caspase- 3 colorimetric kit (Abcam, Cambridge, UK) to measure its activity at 14 days after the induction of SCI. This assay uses a spectrophotometer to measure the absorbance between the wavelengths of 400 and $405 \mathrm{~nm}$ via evaluation of the chromophore $\mathrm{p}$-nitroaniline ( $\mathrm{p}-\mathrm{NA}$ ) after cleavage from a labeled substrate DEVD-p-NA [20].

\section{Enzyme-linked immunosorbent assay}

The levels of tumor necrosis factor- $\alpha$ (TNF- $\alpha)$ and interleukin-1 $(\mathrm{IL}-1 \beta)$ at the site of injury in the spinal cord were measured at 5 days after SCI induction using TNF- $\alpha$ and IL- $1 \beta$ enzyme-linked immunosorbent assay kits (Abcam) according to the manufacturer's protocol.

\section{Histological study}

Three spinal cord tissue specimens were obtained from the animals in each group. After transcardial perfusion with $10 \%$ formaldehyde, specimens were post-fixed, paraffin-embedded, and cut into thin transverse sections. Later, the sections were deparaffinized and stained with Cresyl Violet. The area of tissue damage at the epicenter of the SCI was measured in four tissue sections per rat using ImageJ software (NIH, Bethesda, MD, USA).

\section{Statistical analysis}

The data in the present study are reported as the mean \pm standard error of the mean. GraphPad Prism Software (GraphPad Software Inc., San Diego, CA, USA) was used to analyze the differences among groups using one-way analysis of variance followed by Tukey's post hoc test. A $p$-value of less than 0.05 was considered statistically significant.

\section{Results}

\section{Breast milk stem cell isolation and characterization}

The isolated BMSCs adhered to the plates, and they had a fusiform morphology with a small number of processes (Fig. 1). The results of the flow cytometry analyses showed that the BMSCs did not test positive for endothelial cells (CD144) and hematopoietic lineage (CD34) markers. On the other hand, these cells showed a positive reaction for CD44 and CD105 (Fig. 2).

\section{The Basso, Beattie, and Bresnahan locomotor score}

The rats were examined over a period of 6 weeks to assess the recovery of motor function. The mean $\mathrm{BBB}$ score in the control group was 21 . The control group had significant differences in several parameters compared with the other groups at all postoperative time points throughout the study $(p<0.001)$. After the 6th week, the mean BBB scores for the SCI/Sham group and the BMSC-CM group 


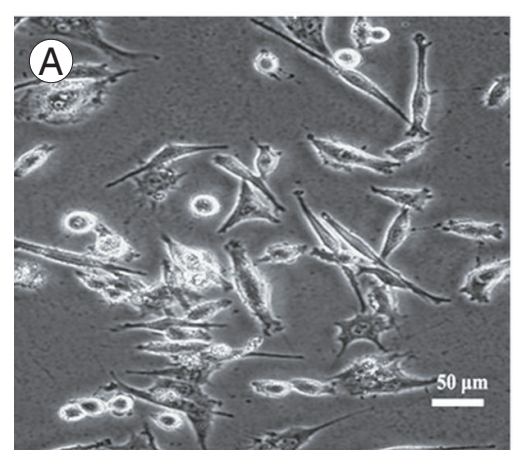

Fig. 1. (A) Breast milk stem cells in the third passage showed a fusiform morphology with a small number of processes. Immunophenotypic features of the breast milk stem cells in passage three. Flow cytometry analysis showed that the isolated and cultured cells were positive for CD44 (B) and CD105 (C) and negative for CD34 (D) and CD144 (E) markers.
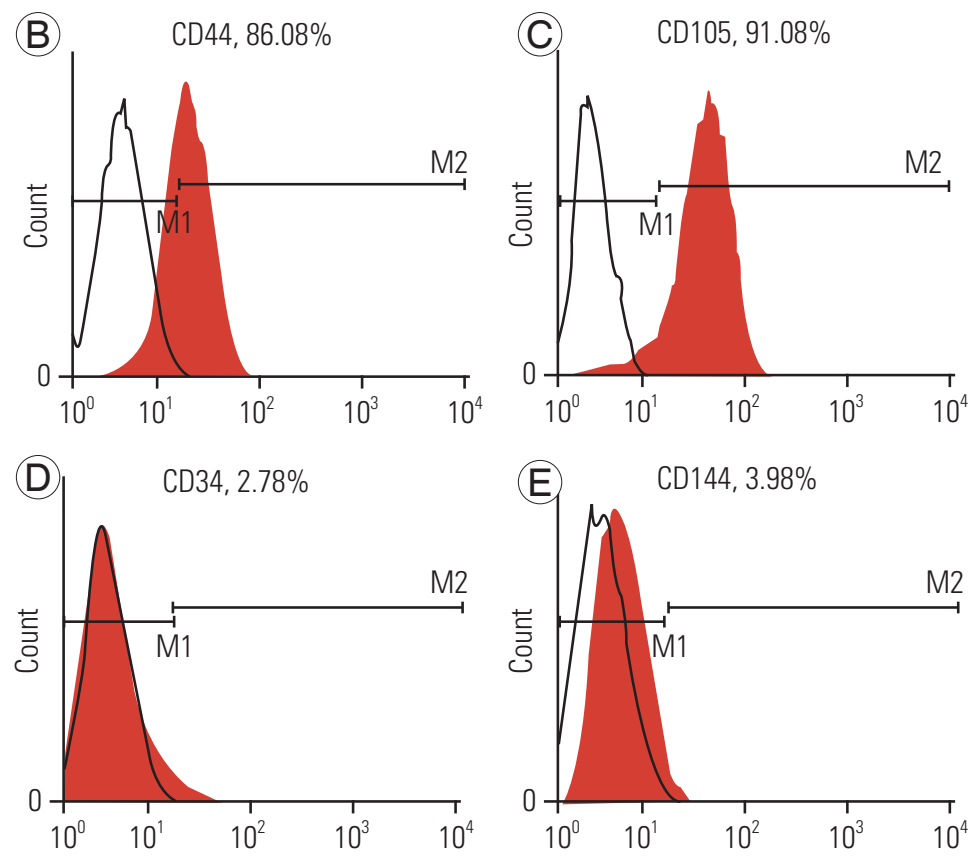

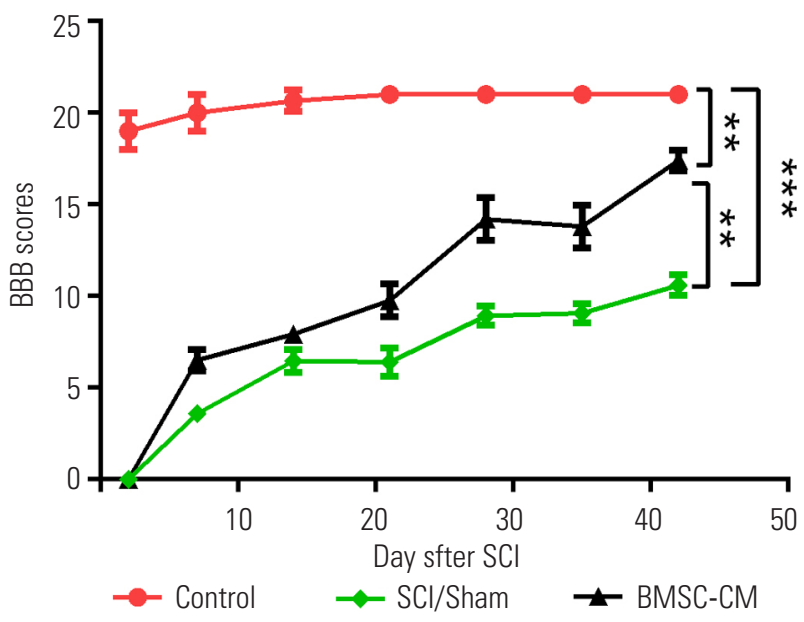

Fig. 2. BBB scores demonstrate locomotor recovery in the different groups during the study. Data show mean \pm standard error of the mean. BBB, Basso, Beattie, and Bresnahan; SCI, spinal cord injury; BMSC$\mathrm{CM}$, breast milk stem cell-conditioned medium. ${ }^{* *} p<0.01 .{ }^{* * *} p<0.001$.

were $10.6 \pm 0.9$ and $17.4 \pm 1.1$, respectively. The group which received BMSC-CM had better neurological outcomes, and there was a significant difference between the BMSCCM group and the SCI/Sham group ( $p<0.01)$ (Fig. 2).

\section{Sensory test score}

Increasing sensitivity to nonpainful, cold stimuli is one of the hallmarks of clinical neuropathic pain. The time required to respond following contact with acetone is inversely proportional to the level of sensitivity. Among all

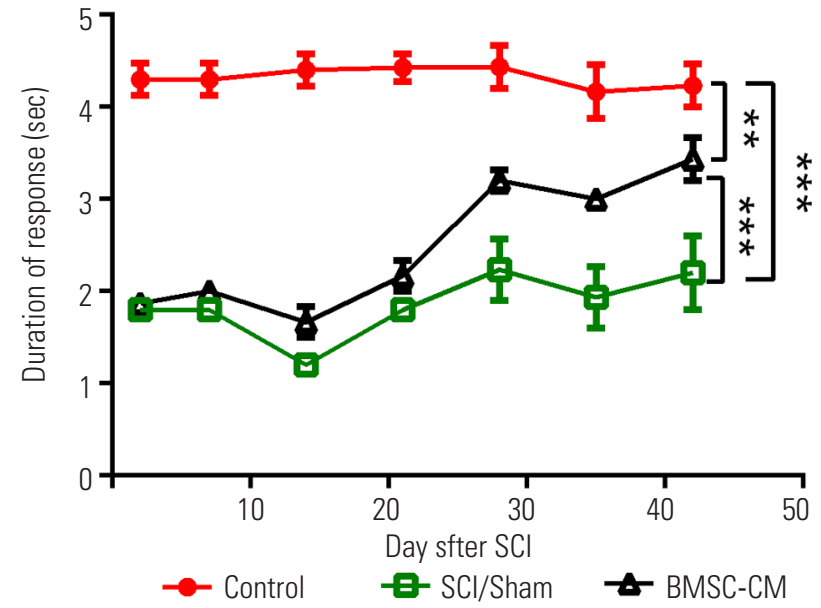

Fig. 3. Duration of response to acetone at different time points of the study in the different groups. Data show mean \pm standard error of the mean. SCI, spinal cord injury; BMSC-CM, breast milk stem cellconditioned medium. ${ }^{* *} p<0.01$. ${ }^{* *} p<0.001$.

groups, the duration of time prior to responding to acetone decreased during the second, third, and fourth weeks after injury. The response time to acetone in the BMSC$\mathrm{CM}$ group at 2, 3, and 4 weeks was significantly reduced compared with the SCI/Sham group $(p<0.001)$ (Fig. 3).

\section{Caspase-3 activity assessment}

To examine apoptosis at the site of injury, we measured the levels of activated caspase- 3 at 14 days after the induction of SCI. The results of the caspase- 3 colorimetric 


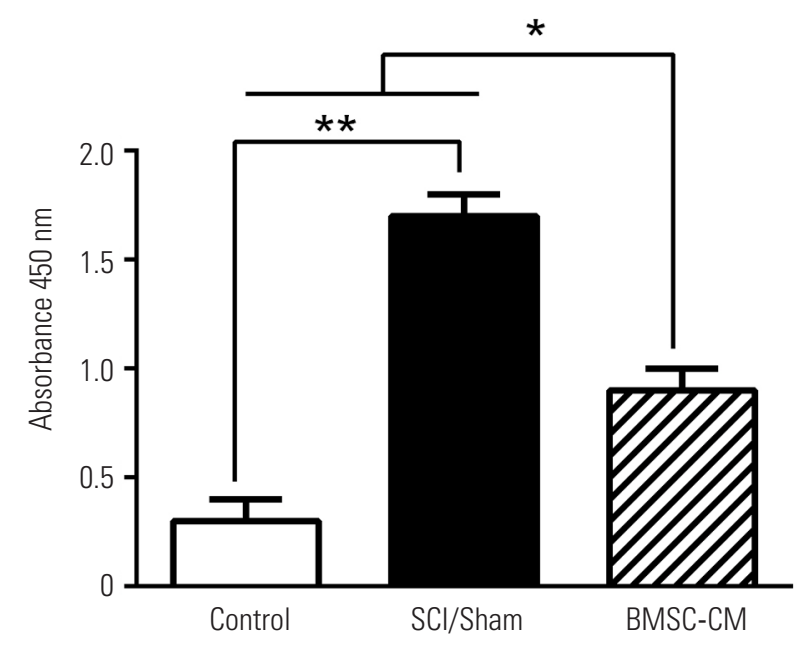

Fig. 4. Caspase 3 activity quantification for apoptosis evaluation. Data show mean \pm standard error of the mean. SCl, spinal cord injury; BMSC$\mathrm{CM}$, breast milk stem cells-conditioned medium. ${ }^{*} p<0.05$. ${ }^{* *} p<0.01$.

assay revealed that SCI increased the activity of caspase-3 in the SCI/Sham group compared with the control group $(p<0.01)$. The light emission of $\mathrm{p}$-NA in the SCI/Sham group was $1.7 \pm 0.1$. By contrast, the activity of caspase- 3 was decreased by BMSC-CM treatment, and this was apparent by the decreased light emission of $\mathrm{p}$-NA in the BMSC-CM group (0.9 $\pm 0.1, p<0.05)$ (Fig. 4 ).

\section{Quantification of proinflammatory cytokines}

The levels of TNF- $\alpha$ and IL- $1 \beta$ were measured at the injured site of the spinal cord at day 5 after the induction of SCI. SCI significantly increased levels of TNF- $\alpha$ and IL- $1 \beta$ in the SCI/Sham group to $310 \pm 14 \mathrm{pg} / \mathrm{mL}$ and $240 \pm 8 \mathrm{pg} /$ $\mathrm{mL}$, respectively $(p<0.01$ versus the control group for both cytokines). In the BMSC-CM group, the levels of TNF- $\alpha$ and IL- $1 \beta$ decreased to $193 \pm 21 \mathrm{pg} / \mathrm{mL}$ and $148 \pm 12 \mathrm{pg} / \mathrm{mL}$, respectively ( $p<0.05$ versus the SCI/Sham group) (Fig. 5 ).

\section{Injury area}

The surface area of the injured tissue was calculated using the Cresyl Violet-stained sections. The control rats did not show damage to the spinal cord tissue. BMSC-CM treatment significantly reduced the area of damage caused by SCI $(p<0.01)$ (Fig. 6).

\section{Discussion}

In the present study, we examined the therapeutic effects
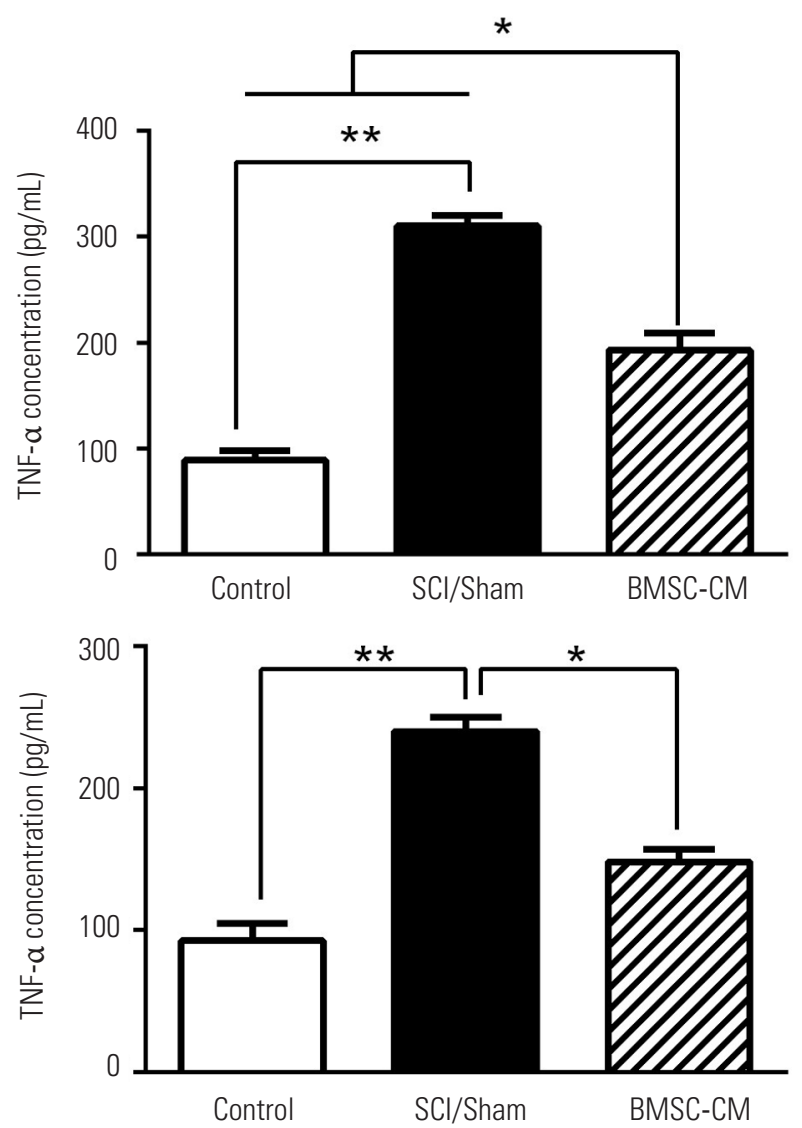

Fig. 5. (A, B) Changes in levels of TNF- $\alpha$ and IL-1 $\beta$. Data show mean \pm standard error of the mean. TNF- $\alpha$, tumor necrosis factor- $\alpha$; IL$1 \beta$, interleukin-1 $\beta$; SCI, spinal cord injury; BMSC-CM, breast milk stem cell-conditioned medium. ${ }^{*} p<0.05 .{ }^{* *} p<0.01$.
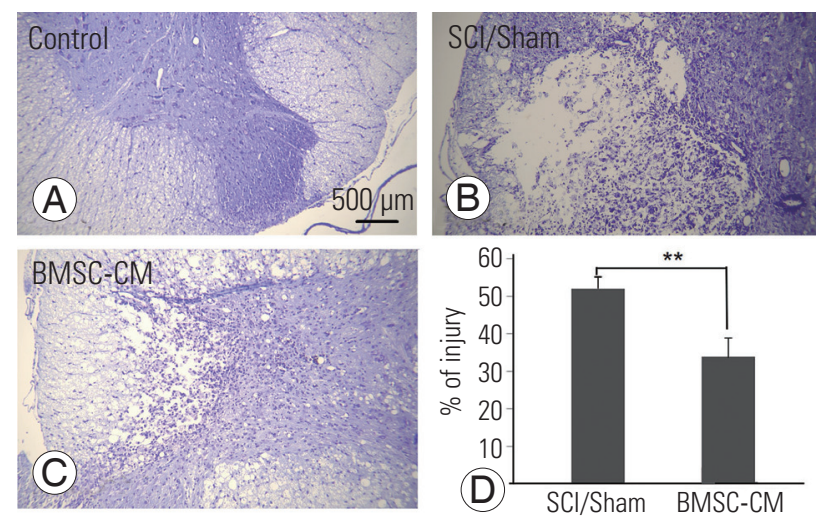

Fig. 6. (A-C) Microscopic images indicate the area of injury among the different groups (Cresyl Violet staining, scale bar=500 $\mu \mathrm{m}$ ). (D) The chart compares the percentage of total surface area that was injured after $\mathrm{SCl}$ among the treatment groups. Data show mean \pm standard error of the mean. SCl, spinal cord injury; BMSC-CM, breast milk stem cell-conditioned medium. ${ }^{* *} p<0.01$.

of BMSC-CM in a rat model of SCI. The results of our study indicate that BMSC-CM can improve the symptoms 
of SCI. After surgery, one common method for treating SCI is the administration of large doses of steroids. However, this treatment has been discontinued due to its timesensitivity, mild results, and the possibility of severe side effects. As a result, alternative treatment strategies are currently being evaluated. Cellular therapies, including Schwann cells, MSCs, embryonic stem cells, and neural stem cells, have been widely investigated for their neuroprotective potential [21]. While cell therapies have shown great progress, they still face certain constraints, including their immunogenicity, cell homing, and loss of differentiation potential. In addition, the micro-environmental cytotoxicity caused by acute SCI presents considerable obstacles against cellular engraftment [22]. Furthermore, some treatments come with important concerns, including painful responses to nonpainful stimuli after neural stem cell transplantation, ethical concerns, or the formation of teratomas after embryonic stem cell therapy [8].

Hosseini et al. [12] reported that BMSCs can differentiate into neural stem cells and neurons. It has also been shown that a population of these cells expresses nestin, which is a marker for neuroectodermal tissue [23]. These features and the ease of isolating BMSC allow these cells to serve as a treatment for nerve tissue injuries. Cell therapies are a promising option for treating diseases, but they still face certain limitations. The survival and differentiation of stem cells within the host tissues remain poor. It has been reported that less than $1 \%$ of MSCs survive for more than 1 week after systemic administration [16]. Loss of differentiation potential, initiation of the host immune response, difficulties in delivery, and poor engraftment of transplanted cells are the major limitations of cell-based therapies $[8,24]$. Since the main effects of MSCs are likely mediated by paracrine mechanisms [25], we examined the effect of BMSC-CM as an alternative treatment for SCI. These experiments demonstrated that BMSC-CM can protect neuronal cells [26,27].

Behavioral recovery after SCI is one of the most significant aims of therapeutic intervention, which may indicate the efficacy of a therapeutic approach [28]. Unlike previous studies that have evaluated the therapeutic potential of CM [29], we found that intrathecal injection of BMSCCM could improve sensory and motor after SCI in rats. Previous studies revealed that SCI causes apoptosis and inflammation in the spinal cord [30]. Likewise, the activity of caspase- 3 was enhanced in the SCI/Sham group. However, BMSC-CM reduced the activity of caspase- 3 after SCI. Hao et al. [31] previously reported that CM from human adipose-derived MSCs inhibits neuronal apoptosis. We showed that the administration of BMSCCM has anti-apoptotic effects in a rat model of SCI [31]. In addition, apoptosis plays a central role in SCI, and it occurs downstream of the inflammatory response $[32,33]$. To evaluate the extent of inflammation, we measured the levels of IL- $1 \beta$ and TNF- $\alpha$ after SCI. The results revealed that the administration of BMSC-CM could also attenuate the production of these cytokines.

\section{Conclusions}

Our data showed that intrathecal administration of BMSC-CM provides therapeutic effects following SCI in rats. We found that BMSC-CM administration improves motor and sensory performance and reduces the production of proinflammatory cytokines such as IL1- $\beta$ and TNF- $\alpha$. Consequently, BMSC-CM reduces apoptosis after SCI. Although the number of rats in each group was too small to draw conclusions, these positive results suggest that the therapeutic mechanisms of BMSC-CM are worth studying in the future.

\section{Conflict of Interest}

No potential conflict of interest relevant to this article was reported.

\section{Acknowledgments}

The authors wish to thank Tehran University of Medical Sciences for financial support of this project.

\section{Author Contributions}

Maryam Borhani-Haghighi: conception and design; data acquisition, and interpretation of data, drafting of the manuscript; Shadan Navid: data analysis, drafting of the manuscript; and Yousef Mohamadi: data acquisition, and interpretation of data.

\section{References}

1. Roselli F, Chandrasekar A, Morganti-Kossmann MC. Interferons in traumatic brain and spinal cord injury: current evidence for translational application. Front 
Neurol 2018;9:458.

2. Yuan J, Zhang X, Zhu R, Cui Z, Hu W. Sparstolonin B attenuates spinal cord injury-induced inflammation in rats by modulating TLR4-trafficking. Mol Med Rep 2018;17:6016-22.

3. Massetti J, Stein DM. Spinal cord injury. In: White JL, Sheth KN, editors. Neurocritical care for the advanced practice clinician. Cham: Springer International Publishing; 2018. p. 269-88.

4. Zhu Y, Uezono N, Yasui T, Nakashima K. Neural stem cell therapy aiming at better functional recovery after spinal cord injury. Dev Dyn 2018;247:75-84.

5. Kane SC, Lewis VR, Conway JE, Garonzik IM. Identification, treatment, and prognosis of geriatric thoracolumbar spinal trauma. In: Rodriguez A, Barraco $\mathrm{RD}$, Ivatury RR, editors. Geriatric trauma and acute care surgery. Cham: Springer International Publishing AG; 2018. p. 101-8.

6. Ahuja CS, Martin AR, Fehlings M. Recent advances in managing a spinal cord injury secondary to trauma. F1000Res 2016;5(F1000 Faculty Rev):1017.

7. Assinck P, Duncan GJ, Hilton BJ, Plemel JR, Tetzlaff W. Cell transplantation therapy for spinal cord injury. Nat Neurosci 2017;20:637-47.

8. Cheng Z, Bosco DB, Sun L, et al. Neural stem cellconditioned medium suppresses inflammation and promotes spinal cord injury recovery. Cell Transplant 2017;26:469-82.

9. Aydin MS, Yigit EN, Vatandaslar E, Erdogan E, Ozturk G. Transfer and integration of breast milk stem cells to the brain of suckling pups. Sci Rep 2018;8:14289.

10. Peterson MB. The presence of stem cells in human breast milk and research implications [dissertation]. Lynchburg (VA): Liberty University; 2016.

11. Witkowska-Zimny M, Kaminska-El-Hassan E. Cells of human breast milk. Cell Mol Biol Lett 2017;22:11.

12. Hosseini SM, Talaei-Khozani T, Sani M, Owrangi B. Differentiation of human breast-milk stem cells to neural stem cells and neurons. Neurol Res Int 2014;2014:807896.

13. Timmers L, Lim SK, Hoefer IE, et al. Human mesenchymal stem cell-conditioned medium improves cardiac function following myocardial infarction. Stem Cell Res 2011;6:206-14.

14. Szekiova E, Slovinska L, Blasko J, Plsikova J, Cizkova D. The neuroprotective effect of rat adipose tissue- derived mesenchymal stem cell-conditioned medium on cortical neurons using an in vitro model of SCI inflammation. Neurol Res 2018;40:258-67.

15. Palomares T, Cordero M, Bruzos-Cidon C, Torrecilla M, Ugedo L, Alonso-Varona A. The neuroprotective effect of conditioned medium from human adiposederived mesenchymal stem cells is impaired by $\mathrm{N}$-acetyl cysteine supplementation. Mol Neurobiol 2018;55:13-25.

16. Tsai MJ, Liou DY, Lin YR, et al. Attenuating spinal cord injury by conditioned medium from bone marrow mesenchymal stem cells. J Clin Med 2018;8:E23.

17. Talaei-Khozani T, Borhani-Haghighi M, Ayatollahi M, Vojdani Z. An in vitro model for hepatocytelike cell differentiation from Wharton's jelly derivedmesenchymal stem cells by cell-base aggregates. Gastroenterol Hepatol Bed Bench 2015;8:188-99.

18. Kay AG, Long G, Tyler G, et al. Mesenchymal stem cell-conditioned medium reduces disease severity and immune responses in inflammatory arthritis. Sci Rep 2017;7:18019.

19. Onifer SM, Rabchevsky AG, Scheff SW. Rat models of traumatic spinal cord injury to assess motor recovery. ILAR J 2007;48:385-95.

20. Borhani-Haghighi M, Kashani IR, Mohamadi Y, Pasbakhsh P. Embryonic intraventricular transplantation of neural stem cells augments inflammation-induced prenatal brain injury. J Chem Neuroanat 2018;94:5462.

21. Donnelly EM, Lamanna J, Boulis NM. Stem cell therapy for the spinal cord. Stem Cell Res Ther 2012;3:24.

22. Tetzlaff W, Okon EB, Karimi-Abdolrezaee S, et al. A systematic review of cellular transplantation therapies for spinal cord injury. J Neurotrauma 2011;28:161182.

23. Fan Y, Chong YS, Choolani MA, Cregan MD, Chan JK. Unravelling the mystery of stem/progenitor cells in human breast milk. PLoS One 2010;5:e14421.

24. Bhang SH, Lee S, Shin JY, Lee TJ, Jang HK, Kim BS. Efficacious and clinically relevant conditioned medium of human adipose-derived stem cells for therapeutic angiogenesis. Mol Ther 2014;22:862-72.

25. Kanekiyo K, Wakabayashi T, Nakano N, et al. Effects of intrathecal injection of the conditioned medium from bone marrow stromal cells on spinal cord injury in rats. J Neurotrauma 2018;35:521-32.

26. Asadi-Golshan R, Razban V, Mirzaei E, et al. Sen- 
sory and motor behavior evidences supporting the usefulness of conditioned medium from dental pulpderived stem cells in spinal cord injury in rats. Asian Spine J 2018;12:785-93.

27. Nakano N, Nakai Y, Seo TB, et al. Characterization of conditioned medium of cultured bone marrow stromal cells. Neurosci Lett 2010;483:57-61.

28. Tator C. Epidemiology and general characteristics of the spinal cord injury patient. In: Benzel EC, Tator $\mathrm{CH}$, editors. Contemporary management of spinal cord injury. Park Ridge (IL): American Association of Neurological Surgeons; 1995.

29. Cantinieaux D, Quertainmont R, Blacher S, et al. Conditioned medium from bone marrow-derived mesenchymal stem cells improves recovery after spinal cord injury in rats: an original strategy to avoid cell transplantation. PLoS One 2013;8:e69515.

30. Gao S, Ding J, Xiao HJ, et al. Anti-inflammatory and anti-apoptotic effect of combined treatment with methylprednisolone and amniotic membrane mesenchymal stem cells after spinal cord injury in rats. Neurochem Res 2014;39:1544-52.

31. Hao P, Liang Z, Piao H, et al. Conditioned medium of human adipose-derived mesenchymal stem cells mediates protection in neurons following glutamate excitotoxicity by regulating energy metabolism and GAP-43 expression. Metab Brain Dis 2014;29:193205.

32. Lan WB, Lin JH, Chen XW, et al. Overexpressing neuroglobin improves functional recovery by inhibiting neuronal apoptosis after spinal cord injury. Brain Res 2014;1562:100-8.

33. Allen AR. Surgery of experimental lesion of spinal cord equivalent to crush injury of fracture dislocation of spinal column: a preliminary report. J Am Med Assoc 1911;57:878-80. 